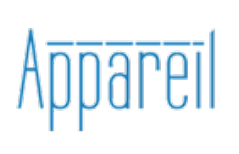

Appareil

11 | 2013

L'espace et l'architecture: état des lieux

\title{
Barrès ou la nationalisation du paysage
}

\section{Alain Brossat}

\section{(2) OpenEdition}

\section{Journals}

Édition électronique

URL : http://journals.openedition.org/appareil/1794

DOI : 10.4000/appareil.1794

ISSN : 2101-0714

Éditeur

MSH Paris Nord

\section{Référence électronique}

Alain Brossat, « Barrès ou la nationalisation du paysage », Appareil [En ligne], 11 | 2013, mis en ligne le 26 septembre 2013, consulté le 30 juillet 2020. URL : http://journals.openedition.org/appareil/1794 ; DOI : https://doi.org/10.4000/appareil.1794

Ce document a été généré automatiquement le 30 juillet 2020.

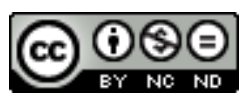

Appareil est mis à disposition selon les termes de la Licence Creative Commons Attribution - Pas d'Utilisation Commerciale - Pas de Modification 4.0 International. 


\title{
Barrès ou la nationalisation du paysage
}

\author{
Alain Brossat
}

1 On ne lit plus guère Maurice Barrès aujourd'hui, non sans motif, ce qui ne veut pas dire qu'il n'y aurait plus aucun intérêt à s'y replonger de temps à autre. C'est ce que je vais essayer de montrer en vous proposant une lecture de Colette Baudoche, histoire d'une jeune fille de Metz $z^{1}$ inspirée par l'intitulé de ce colloque.

2 Ce roman est publié par Barrès en 1909, quelques années avant le grand cataclysme, dont on ne peut pas dire, au reste, qu'il véhicule le pressentiment. On pourrait penser que le souvenir en est passé aux pertes et profits de la Grande Guerre, tant la fable kitsch qu'il présente a été balayée par la tempête qui a soufflé sur l'Europe entre 1914 et 1918. Mais les choses sont un peu plus compliquées. Le Silence de la mer de Vercors², publié clandestinement par les éditions de Minuit en 1942, est, très explicitement, une réponse au roman de Barrès dont il emprunte, grosso modo, la trame narrative. Et le film moyen métrage de Jean-Marie Straub et Danièle Huillet, Lothringen!, Lorraine en allemand (1994), est une libre adaptation de Colette Baudoche. Sous l'oubli « littéraire », donc, le cheminement d'une mémoire souterraine, celle des complexités équivoques de la relation franco-allemande.

3 L'histoire est simple. Colette Baudoche et sa grand-mère, Lorraines de souche, vivent à Metz au temps de l'annexion de la province par l'Allemagne, suite à la défaite de 1870 . Elles sont conduites par la nécessité économique à prendre en pension un professeur allemand venu de Kœnigsberg pour prendre part à la germanisation de l'enseignement en Lorraine annexée, le $\mathrm{D}^{\mathrm{r}}$ Frédéric Asmus. Tout imbu des préjugés du vainqueur au début, mais rapidement attiré par la fraîche simplicité de la jeune Colette, impressionné par l'inébranlable fermeté patriotique des deux femmes, le professeur et occupant va bientôt subir l'ascendant de l'occupé et se dégrossir à son contact. Ses mœurs s'adoucissent, il tombe sous le charme du tempérament et du paysage lorrain, bref voici le colonisateur captivé par le colonisé. À la fin, ayant rompu ses fiançailles avec la Walkyrie teutonne (sic) auprès de laquelle il s'est engagé avant de partir pour la Lorraine, il demande la main de Colette. Mais celle-ci, même si elle n'est nullement 
insensible à sa gentillesse et à ses efforts pour se rapprocher de l'âme lorraine, refuse, dans un sursaut patriotique : il y a, entre elle et lui, tous ces morts qui lui interdisent de conclure, avec l'occupant, une « fausse paix » matrimoniale...

4 Ce qui constitue le trait proprement barrésien, dans ce roman comme dans d'autres, c'est l'application et la constance avec laquelle la fable est enracinée dans une topographie, reconduite à la terre, aux lieux, à l'espace, aux paysages. Ce que l'ennemi ne saurait s'approprier, ce qui échappe résolument à ses prises, c'est cette géographie habitée, car chaque monument, chaque colline, chaque vignoble, chaque village porte la marque d'un génie autochtone qui résiste à son emprise et auquel en tant qu'étranger, semi-barbare venu de l'Est, il ne saurait être, d'emblée du moins, sensible. En ce sens, décrire un paysage de la Lorraine occupée, évoquer Metz et ses monuments, c'est déjà faire une profession de foi patriotique, et résister à l'occupation. Ainsi, cette phrase sur laquelle s'ouvre le roman :

Il n'y a pas de ville qui se fasse mieux aimer que Metz. Un Messin français à qui l'on rappelle sa cathédrale, l'Esplanade, les rues étroites aux noms familiers, la Moselle au pied des remparts et les villages disséminés sur les collines, s'attendrit. (p. 11)

Ou bien encore, ce passage, exemplaire de ce style classique, mièvre et facile, un style de guide touristique patriotique - l'anti-Baedecker ! :

Depuis l'Esplanade, on devine sous un ciel nuageux douze villages vignerons, baignés ou mirés dans la Moselle, et qui nous caressent, comme elle, par la douceur mouillée de leurs noms: Scy, qui donne le premier de nos vins; Rozérieulles, où chaque maison possède sa vigne; Woippy, le pays des fraises; Lorry, que ses mirabelles enrichissent ; tous chargés d'arbres à fruits qui semblent les abriter et les aimer. (p. 13)

Éden à l'Est, donc, et où tout l'atteste : ici, c'est la France! Un espace et un paysage où chaque détail a valeur de signature patriotique.

6 Ce qui est intéressant dans la conception barrésienne du paysage, telle qu'elle se présente dans ce roman, c'est qu'elle abolit radicalement l'opposition traditionnelle entre l'espace et le temps, ceci dans la mesure même où un paysage, pour lui, c'est avant tout un dépôt d'histoire et de civilisation, c'est-à-dire un lieu à la fois d'exposition (de présentation) et de décantation, de stratification d'un passé en tant que celui-ci est l'œuvre et la marque spécifique (la signature, une fois encore) d'une communauté. Cette notion du topos comme espace d'inscription d'une singularité historique et culturelle s'énonce très distinctement dans ce passage où Barrès entend montrer comment la présence allemande défigure le paysage messin et celui de la région : « $\mathrm{Ce}$ pays était épuré, décanté [je souligne], je voudrais dire spiritualisé ; ils [les occupants] le troublent, le surchargent, l'encombrent, ils y versent une lie » (p. 22).

7 Recourant à un procédé proto-cinématographique, Barrès nous livre une description de Metz « reconstruite à l'allemande et selon les besoins du vainqueur », une description comme filmée d'avion, en surplomb, avec la gare et son style colossal (Barrès nous épargne le «k », mais le cœur y est...), bref, écrit-il, « on croit voir, [dans ce paysage urbain profané par l'invasion] figées en saindoux, les folies d'étudiants architectes à la taverne d'Auerbach» (p. 20).

8 L'occupation allemande de la Lorraine, c'est donc d'abord cela: une profanation du paysage, une grossière surcharge de ce qui en fait une inimitable signature. Mais le paysage n'est pas inerte, il est un être vivant, il a une âme et il se défend contre cet accaparement et ces défigurations, il refait obstinément surface sous le grossier vernis 
teuton: les vieilles parties de Metz, écrit Barrès, «nous ramènent vers la France » et « là-bas, c'est le synonyme le plus fréquent de l'idéal ».

Le locataire des dames Baudoche, le $\mathrm{D}^{\mathrm{r}}$ Asmus, n'est pas un mauvais bougre, il est disposé à tirer parti de son séjour à Metz pour perfectionner son français, il est partisan d'une occupation tempérée et se sépare de ceux qui préconisent une germanisation à outrance de la Lorraine annexée - mais il n'en demeure pas moins un balourd, comme tous ceux de son espèce : il s'habille à la mode de Konigsberg, se nourrit de bière et de charcuterie, pose des questions indiscrètes aux dames Baudoche, prend la mouche si son petit déjeuner lui est servi avec une minute de retard - bref, il est, comme disent ces dames, " un animal de la grosse espèce ». Et, ce n'est pas un hasard, c'est encore le paysage qui est le meilleur révélateur, la meilleure surface de réfraction de ce déficit en matière de mœurs et de culture. Un dimanche matin, peu après son installation à Metz, Asmus s'en va faire une excursion à pied du côté du village de Scy avec quelques collègues allemands. Ils cheminent parmi les vignes et les vergers, croisent des paysans qui parlent français entre eux et s'en étonnent, ils montent vers une terrasse d'où ils découvrent un riant panorama :

Devant eux s'étendait un pays à la mesure humaine, vaste sans immensité, façonné et souple, et, près de sa rivière, Metz, toute plate au ras de la plaine, et que spiritualise le vaisseau de sa haute cathédrale. Cette fin de septembre est l'époque la plus charmante de la Lorraine. Peu de pluie, du vent rarement, une température stimulante et les vignes à la veille d'une joyeuse vendange. Ce matin-là, le ciel, les miroirs d'eau, les prairies composaient un de ces paysages d'automne lorrain où les couleurs les plus éblouissantes d'argent et de vert s'harmonisent pour nous procurer un long repos de rêverie. (p. 50-51)

Et là, ça casse :

Ils [les professeurs allemands] n'en comprirent pas la délicatesse et s'accordèrent à proclamer qu'ils avaient dans la vieille Allemagne de plus grands paysages [je souligne]. (p. 51)

Et comme pour enfoncer le clou de ce trait grotesque, le narrateur ajoute, de crainte sans doute que nous n'ayons pas bien compris :

Il manquait à ces jeunes gens, venus d'un ciel où la Walkyrie chevauche les nuages,

d'avoir été élevés à sentir qu'il y a dans la simplicité de notre nature une suprême élégance. (p. 51-52)

(C'est ça, le kitsch barrésien : cette façon de mettre les points sur les $\mathrm{i}$, de faire du pathos, de " charger ", comme les mauvais acteurs).

Bref, ce qui caractérise peut-être en premier lieu ces envahisseurs comme des barbares, même s'ils se conduisent dans la Lorraine annexée, avec une certaine circonspection, c'est leur incapacité d'être sensibles à ce qui, dans ce paysage, témoigne de la présence vivante d'une vieille civilisation. Leur seul critère est le grand, en tant que celui-ci est supposé témoigner de la puissance. Ils ne méritent pas la beauté de ce paysage, sa délicatesse, tout ce qui, dans la dimension de l'impalpable, rend compte et du génie du peuple qui l'habite et de la belle unité que compose celui-ci avec son environnement la colline sur laquelle s'est niché le village, le clocher émergeant à l'horizon, la vigne descendant en pente douce sur la rivière... Tout, dans ce paysage, accuse la violence que lui fait subir leur présence pesante, indiscrète, déplacée.

11 Simplement, il se trouve que le $\mathrm{D}^{\mathrm{r}}$ Frédéric Asmus n'est pas entièrement taillé dans le bois dont est faite cette occupation imbue d'elle-même, aussi prussien soit-il. Une certaine forme de disponibilité ou d'ouverture de tempérament va progressivement le rendre sensible à l'urbanité des dames Baudoche, à l'«agrément d'une certaine 
supériorité d'hygiène et de goût » qu'il rencontre dans leur logis, à « la douceur de l'eau bruissante et des voix traînantes qui parlent français » (la rivière, l'eau et les voix, c'est tout un). Certes, il ne sait pas encore "distinguer les âmes du pays messin », mais quelque chose en lui commence à être affecté par cet environnement où «l'harmonie des objets matériels avec leur sens moral est parfaite ", bref, c'est la fable antique qui revient : le colonisateur romain (prussien) est captivé (civilisé) à son corps défendant par le colonisé grec (lorrain). Les dames Baudoche vont être ses guides, elles vont l'initier à cette civilisation lorraine dont il ignore les fondements et dont les subtilités le dépassent. Elles lui exposent avec autant de fermeté que de délicatesse tout le tort que cause à leur pays l'occupation dont il est l'un des agents. Mieux que cela, elles entreprennent de le convertir aux principes de cette grande civilisation et, de maitre qu'il était, le voici transformé en disciple.

Vous anéantissez, [lui disent en substance ses interlocutrices] des aspects qui sont liés à toutes nos vénérations. Vous coupez les arbres et comblez les puits de notre Lorraine morale. Et les formes que vous construisez, nous n'y avons pas de place. [...] M. Asmus écoutait, bouche bée, comme il aurait suivi le cours de quelque maître autorisé. Il entrevoyait une civilisation nouvelle pour lui, et toute fière [je souligne]. (p. 76)

Tout se tient : en même temps qu'elles corrigent ses fautes d'accent et de grammaire, qu'elles dégrossissent ses mœurs de table, les dames Baudoche apprennent au Prussien à voir la Lorraine, au fil de leurs promenades dominicales et de leurs étapes dans les auberges de village. C'est à des principes de civilisation qu'il est initié, en bloc.

Tout comme, chez Barrès, l'histoire et la géographie font bloc: il y a cette présence vivante des aïeux et cette fidélité aux morts, cet enracinement de l'identité propre dans le passé qui empêche à jamais Colette de se sentir allemande, de se voir en Allemande. Mais cette dimension généalogique est indissociable, bien sûr, du lien à la terre, de l'enracinement dans le terroir, lequel s'atteste par la capacité de se situer dans l'environnement, de voir le paysage. Double enracinement, donc, si bien que l'on pourrait parler, à propos de Barrès, d'un patriotisme écologique, d'un écolo-patriotisme lorrain (au moins autant que français, en l'occurrence, le petit pays d'abord, la nation ensuite!), on le voit bien quand il parle de l'économie morale de sa Lorraine en évoquant des « arbres coupés », des " puits comblés ».

13 Si quelque chose devait survivre à la débâcle du kitsch barrésien, ce serait peut-être cet élément: l'invention du paysage comme motif identitaire, patriotique, comme économie morale, dans sa relation indéfectible à une histoire partagée. L'idée (que l'on va retrouver chez les Straub) que non seulement le paysage a une histoire, mais qu'il est histoire, qu'il est, tout simplement, un autre visage de l'Histoire, avec une majuscule.

L'inclination qui porte le professeur vers Colette va l'aider à se rapprocher de l'esprit de ces lieux où il demeure un intrus, un «immigré » comme dit le narrateur. Elle va l'aider à secouer le «fatras poussiéreux [qui] surcharge les greniers de son esprit». Ainsi, lorsque de retour d'une visite à Nancy, en Lorraine demeurée française et qui l'a laissé émerveillé, il trouve Metz toute animée de la récente décision prise par l'autorité locale de supprimer l'enseignement du français dans les écoles de quatre villages, Moyeuvre-Grande, Fontoy, Knutange et Audun-le-Tiche, une décision qui scandalise la population locale et dont se réjouissent ses collègues allemands, il se sépare de ces derniers, soutenant que « détruire la langue française en Lorraine, c'[est] bel et bien détruire des intelligences ». Mais il fait mieux : il s'immerge dans ce paysage où se trouve toute entière sertie la vérité irrécusable de ce petit pays, allant de village en 
village, entrant dans les maisons, parlant avec les gens afin de vérifier leur enracinement dans la langue, la culture, le tempérament français. Il découvre «la campagne autour de Metz [...] infiniment chargée, nuancée, pétrie par la culture, par les hommes, par des siècles de grande histoire et d'obscure activité » (p. 149). Tout ceci affecte, impressionne notre Prussien qui se voit déambulant au milieu d'un «noble tableau de Poussin ». Il découvre aussi ce qu'est la qualité morale d'un paysage, pour autant que la beauté de celui-ci (par opposition au pittoresque) renvoie toujours à la qualité de la race qui le peuple. Ayant esquissé une comparaison un peu audacieuse entre cette campagne lorraine et la Toscane, le narrateur précise :

Toutefois, l'Arno toscan n'a pas la noblesse fière, la chasteté de notre rivière, quand les saules courbés par le vent se penchent sur elle, qui fuit dans les prairies sombres. Et les larges couleurs profondes que notre terre prend parfois le soir s'accordent avec les vertus éprouvées et calmes de notre nation [je souligne]. (p. 150)

Ce que découvre Frédéric Asmus, ce à quoi il est appelé à se convertir, c'est l'immémorial qui gît au fond d'un paysage, l'immuable, ce qui, en dépit des soubresauts et des violences de l'Histoire, persévère dans son être : ces villages qui «tous gris sous des toits rouges, n'ont pas changé depuis des siècles ». L'immémorial qui se dévoile dans ce paysage, avec l'inévitable mélancolie qu'il attise, descelle le Prussien de son socle nationaliste, germaniste, quand bien même il ne serait pas en mesure de recueillir la meilleure part de l'héritage qui s'y attache :

Avec quelle avidité, en marchant seul dans la campagne, il regarde, écoute, admire ce qui naît spontanément du sol! Comme il se réjouit d'avoir tant d'inconnu à approfondir! La place Stanislas lui a délivré, épuré l'esprit [lors de sa visite de Nancy, donc] ; cette campagne lui émeut le cœur. (p. 153-154)

Cette campagne recèle des puissances insoupçonnées: elle fait son éducation sentimentale, morale, culturelle, historique...

Dès lors, la fable du roman patriotique de Barrès se trouve à deux doigts de se retourner, de se renverser en son contraire exactement - en éloge de l'interculturalité franco-allemande, avec un demi-siècle d'avance, donc, et en effaçant sur l'ardoise magique de l'histoire les deux guerres mondiales. Écoutons attentivement cette phrase qui nous conduit sur le bord de ce renversement :

Il y a des petits villages, isolés au milieu des espaces ruraux, qui, le soir, à l'heure où l'on voit rentrer les bêtes et les gens, m'apparaissent comme des gaufriers (sic), et je crois que tout être, fût-il barbare prussien, soumis à leur action patiente et persistante, $y$ deviendrait lentement Lorrain. (p. 154-155) [je souligne]

Et Barrès d'insister sur la force d'attraction irrésistible de ce paysage : l'activité de ceux qui l'ont façonné s'est faite "ce groupe de maisons, ce clocher, cet abreuvoir, cette école qu'entourent les champs bigarrés de couleurs et de formes, et si l'on entre dans cette communauté [je souligne], on y vient nécessairement se conduire et penser comme ont fait les prédécesseurs. [...] C'est un jardin de Paradis, et l'homme de la Prusse orientale ne songe pas à le nier» (p.155-156). Il tombe amoureux de ce paysage et de ceux qui l'habitent :

il reconnaît chez les vieilles Lorraines, sous leurs bonnets gaufrés, non pas une âme meilleure et plus limpide que l'âme des vieilles mamans allemandes, mais une vive et saine malice ; il aime à voir les charmantes figures, déjà militaires, des enfants de quatorze ans, auprès des figures paisibles et claires de leurs grands-parents; il écoute avec un plaisir de sympathie, çà et là, dans les champs, mêlé aux mots que les paysans disent aux chevaux l'accent railleur et gentil des jeunes filles, de qui la halette, sous l'immense soleil, voile la figure [...] À défaut d'une affection de naissance, c'était presque un amour de mariage. Il découvrait, créait, mûrissait en 
lui une Lorraine par à peu près. Il la composait assez bizarrement d'un amalgame de ses rêves avec les notions que ses logeuses lui fournissent. [...] le jeune Germain [...] voit naître dans son esprit une parenté avec les gens qui façonnèrent cette campagne. (p. 156-159)

17 Comme on le voit très clairement ici, toutes les conditions culturelles sont remplies pour que ces marches, ces espaces interstitiels, ces paysages capables de captiver le cœur d'un natif de la lointaine Kœnigsberg deviennent le théâtre de la rencontre des deux civilisations, mieux, de leur amalgame - comme c'est bien le cas aujourd'hui dans tous les espaces transfrontaliers de l'Alsace et la Lorraine. Mais il se trouve que cette belle fable est surplombée, comme dans la tragédie grecque, par le décret d'une puissance divine dont l'élément est la démesure - ici la concurrence des États-nations. Hybris et Nemesis vont en bateau et quelques millions de morts français et allemands plus loin, les rues de Strasbourg peuvent enfin être envahies par les touristes allemands tous les week-ends, en été, sans que même les Barrès d'aujourd'hui (M. Montebourg, M. Chevènement...) n'y puissent voir un grand malheur - ça fait marcher le commerce.

L'impardonnable, dans le roman de Barrès, se situe précisément ici : dans la volte-face de celui qui, après avoir écrit les lignes que je viens de lire, se fait l'agent de la nemesis historique, de l'Histoire comme idole cannibale, en interdisant à Colette de céder à son penchant pour ce bon bougre de Frédéric Asmus... On se rappellera ici que le 13 mai 1921, à l'occasion d'un procès solennel monté par Dada et les surréalistes, Barrès fut accusé à bon escient, au vu de la catastrophe échue, de «crime contre la sûreté de l'esprit ».

19 Reprenons donc, pour aller vers l'inexorable conclusion. D'étroites correspondances s'établissent, dans le cœur du jeune professeur, entre ce que lui dévoile le paysage lorrain et ce qu'il découvre sur le visage de la jeune Colette : «Son émotion, qui cherche un objet vivant, se rassemble sur la jeune Messine. Il songe qu'après une averse, en été, la lumière sur les prairies a la jeune noblesse du regard de Colette, émue des malheurs de sa nation" (p.215). Le visage de Colette est un paysage qui porte les marques douloureuses de l'Histoire, comme la campagne lorraine qu'il parcourt en compagnie de ses hôtesses et où il découvre, comme autant d'affreuses cicatrices défigurant la "gracieuse civilisation lorraine", les souvenirs des tragédies franco-allemandes. Le plateau de Gravelotte, les châteaux lorrains tombés aux mains des Allemands et défigurés par les rénovations intempestives, etc. Il entend les dames Baudoche lui raconter comment, en septembre 1872, cette riante campagne fut parcourue par les colonnes ininterrompues de réfugiés qui, refusant de devenir allemands, abandonnaient tout pour passer en Lorraine française... Bref, placé à si bonne école, il bascule inexorablement du côté de la civilisation la plus raffinée, celle qui lui a enseigné à se tenir à table, à identifier dans ce pays cette " unité de style » qui en fait une nouvelle Grèce ; involontairement, il met en regard cette civilisation avec celle dont il est issu, si mal dégrossie, il se querelle à ce propos avec ses collègues pangermanistes et, ajoute avec finesse le narrateur, « il compare cette petite Colette aux Gretchen de chez lui »- ayant donc rompu ses fiançailles avec sa fiancée du grand Est, il tente d'embrasser Colette qui feint de s'en indigner mais serait bien près de se laisser tenter et qui s'interroge : «Après trente-cinq ans, est-il excusable d'épouser un Allemand ?»

Et puis qui, après avoir balancé pendant un mois, se ressaisit et tranche: elle "reconnaît l'impossibilité de transiger avec ces morts qui sont là présents ». Entre M. Asmus et elle, « ce n'est pas une question personnelle, mais une question française ». 
L'ayant dûment convoqué, elle lui fait donc cette solennelle déclaration : «Monsieur le docteur, dit la jeune fille, je ne peux pas vous épouser. Je vous estime, je vous garderai une grande amitié ; je vous remercie pour le bien que vous pensez de nous. Ne m'en veillez pas ». Et le narrateur d'ajouter : «Le Prussien s'incline sèchement, et s'éloigne ; il va réfléchir, des mois et des mois, pour savoir s'il doit admirer ou détester cette réponse ». (p. 255-256) Encore et toujours, en dépit de tout, cette indécrottable lenteur d'esprit du Prussien...

Au fond, le coup de force du narrateur idéologue patriotique consiste à transformer in extremis le paysage qui, tout au long du récit, nous a été présenté comme le milieu de la conciliation, celui de l'apprivoisement de l'occupant, de l'adoucissement de ses mœurs, condition d'une heureuse acculturation, en tout autre chose: nécropole, champ d'honneur, c'est-à-dire lieu de mémoire de l'esprit de revanche. Un rêve funèbre et glorieux qui se projette vers l'avant puisque c'est en effet ce qu'est devenu et est aujourd'hui encore ce paysage constellé des cimetières militaires et autres sites commémoratifs des grands carnages de la première guerre mondiale. Mémoire plus vivante que jamais, puisqu'à l'orée des commémorations du centenaire du commencement de la Grande Guerre, je suis, ici, bien fondé à en vouloir personnellement à ce con de Barrès d'avoir contribué par ses moyens propres à pourrir la vie de mes deux grands-pères plongés l'un et l'autre dans cette tourbe sanglante, quatre années durant.

Mais tentons, puisque nous échoit ici la grâce de la naissance tardive, d'être équitables : cet écrivain médiocre, incroyablement surestimé de son vivant, pour de fort mauvaises raisons sans doute, cet antidreyfusard spasmodique, ce chauvin kitsch pourrait peutêtre, être repêché en appel in extremis à la faveur d'un tout petit détail : avec tous ses travers, il savait regarder un paysage. Or, s'il est, à notre époque, une capacité qui s'est perdue, je l'observe tous les jours sur nos contemporains, c'est bien celle-ci. Or, le paysage, c'est ce qui nous donne accès à la vibration de l'Être, là où nos existences croulent sous le fardeau des étants. Ergo, pour renchérir sur le jugement des surréalistes, Barrès est un con, certes, mais il demeure constant que nous pouvons, parfois, tirer quelque bénéfice de la fréquentation d'un con, voire de plusieurs...

\section{NOTES}

1. Maurice Barrès, Colette Baudoche, histoire d'une jeune fille de Metz, Paris, Juven, 1909.

2. Vercors, Le Silence de la mer, Paris, Minuit, 1942. Rédigé au cours de l'été 1941 et publié clandestinement en février 1942. 


\section{RÉSUMÉS}

Dans son roman Colette Baudoche (1909), Maurice Barrès tente de mobiliser ses lecteurs au service de la revanche en racontant l'histoire d'une jeune fille lorraine qui refuse d'épouser un professeur allemand, au temps de l'annexion allemande qui suit la défaite de 1870. L'évocation du paysage, considéré comme émanation du génie d'un peuple, occupe, dans ce roman, une place considérable. C'est cette «nationalisation », cette idéologisation patrimoniale du paysage lorrain qui sont étudiées dans ce texte.

\section{INDEX}

Mots-clés : Lorraine, lorrain, paysage, Barrès, guerre, nation, nationalisme 